\title{
Radiofrequency catheter ablation of accessory atrioventricular pathways: primary failure and recurrence of conduction
}

\author{
B Xie, S C Heald, A J Camm, E Rowland, D E Ward
}

\begin{abstract}
Objective-To identify possible factors associated with primary failure of radiofrequency ablation of accessory pathways or recurrence of accessory pathway conduction.

Patients and methods-Radiofrequency ablation of accessory pathways failed in $\mathbf{2 5}$ of 243 patients, and recurrence of accessory pathway conduction occurred in an additional 13 patients. Factors possibly related to primary failure and recurrence were analysed.

Results-Primary failure and recurrence were less frequent in patients with left sided pathways $(7 \% v 19 \% ; 4 \% v 24 \% ; P=$ $0 \cdot 04)$. The factors that might relate to primary failure included an unstable catheter position (seven patients), a possible epicardial pathway (six patients), or misdiagnosis of accessory pathway location (two patients). The major factors for recurrence included the stability of the local atrial electrogram $\leqslant 0.5$ together with the stability of the local ventricular electrogram $\leqslant 0.8$, and prolonged time to pathway conduction block $\geqslant 12$ seconds). Thirty one patients underwent repeat ablation which was successful in 28 .

Conclusions-Primary failure and recurrence were more frequent in patients with right sided pathways. An unstable catheter position and a possible epicardial pathway location are the main contributing factors for primary failure, while unstable local electrograms and prolonged time to block are independent predictors for recurrence.
\end{abstract}

(Heart 1997;77:363-368)

Keywords: radiofrequency catheter ablation; accessory pathway; low energy direct current

Department of

Cardiological

Sciences, St George's

Hospital Medical

School, London

B Xie

S C Heald

A J Camm

E Rowland

D E Ward

Correspondence to:

Dr D E Ward, Department

of Cardiological Sciences,

of Cardiological Scien

St George's Hospita
Medical School,

Cranmer Terrace,

Cranmer Terrace,

Kingdom.

Accepted for publication

14 January 1997
Radiofrequency catheter ablation is now first line treatment in symptomatic patients with accessory atrioventricular pathways owing to its high efficacy and low risk. Previous studies have shown that the elimination of accessory pathway conduction can be achieved in $>90 \%$ patients. ${ }^{12}$ However, in some patients accessory pathway conduction cannot be abolished even after a lengthy procedure, and in other patients pathway conduction recurs after may predispose to failure and recurrence have not been well defined. The objective of this apparently successful ablation. Factors that study was to analyse possible factors associated with primary failure to abolish accessory pathway conduction, or recurrence of accessory pathway conduction after an initially successful procedure.

\section{Methods}

STUDY POPULATION

From January 1991 to August 1994 a consecutive series of 243 patients with 248 accessory pathways underwent radiofrequency catheter ablation. One hundred and fifty nine patients were male and 84 female, age range 6 to 71 years, mean 34 (SD 17) years. Each patient had recurrent episodes of atrioventricular reentrant tachycardia, atrial fibrillation, or both. Most patients had failed treatment with several antiarrhythmic drugs, either because of lack of efficacy or because of unacceptable side effects. Structural heart disease was present in nine patients (cardiomyopathy in four, Ebstein's anomaly in three, mitral stenosis in one, and mitral valve prolapse in one).

RECORDING AND ANALYSIS OF LOCAL

ELECTROGRAMS

We analysed the endocardial electrograms. These included the interval from onset of the earliest delta wave to local ventricular electrogram (ORS-V), the local atrioventricular interval (AV), the local ventriculoatrial interval (VA), the stability of the local atrial and ventricular electrograms, and the presence of a possible accessory pathway potential, recorded at successful sites (permanent abolition of accessory pathway conduction), at sites where block in accessory pathway conduction was only transient, or the best electrograms obtained during failed procedures. The details of determination of local electrocardiogram characteristics have been described previously. ${ }^{3}$ The stability of local atrial and ventricular electrograms was defined as the ratio of the minimum to maximum amplitudes of the five complexes immediately preceding radiofrequency current delivery. No attempt was made to verify possible accessory pathway potentials by programmed stimulation.

\section{ELECTROPHYSIOLOGICAL STUDY AND}

\section{CATHETER ABLATION}

Electrophysiological studies had been performed in a separate session in 40 patients, and immediately before catheter ablation in all patients. The protocols for standard electrophysiological studies and radiofrequency ablation have been described elsewhere. ${ }^{34}$ In brief, 
ablation was attempted with a $7 \mathrm{~F}$ deflectable catheter with a $4 \mathrm{~mm}$ tip and $2.5 \mathrm{~mm}$ interelectrode spacing inserted into the femoral vein, femoral artery, or subclavian vein. After precise mapping, radiofrequency current was delivered between the distal electrode of the ablation catheter and a large skin electrode, using a $500 \mathrm{kHz}$ generator (HAT 200s, Dr Osypka $\mathrm{Gmbh}$ ) at constant preset electrical power (usually $25-35 \mathrm{~W}$, range 15-50 W) for 30 seconds. If conduction in accessory pathway failed to block within 10 seconds (occasionally longer), energy delivery was interrupted. After initially achieving successful interruption of accessory pathway conduction patients remained in the laboratory for between 20 and 60 minutes to see if accessory pathway conduction would recur. Atrial pacing, ventricular pacing, or both were performed immediately after ablation and 20 to 60 minutes after the last radiofrequency application.

Left sided accessory pathways were approached retrogradely through the aorta, or transeptally. Right sided accessory pathways were approached by positioning the ablation catheter on the tricuspid valve annulus through the femoral or subclavian vein. Posteroseptal accessory pathways were approached by positioning the ablation catheter on the medial aspect of the mitral annulus retrogradely through the transaortic route, or on the tricuspid annulus or within the proximal coronary sinus through the femoral vein.

Primary failure was defined as persistence of conduction in the accessory pathway at the time of leaving the catheter laboratory following the first ablation session. Recurrence was defined as successful abolition of accessory pathway conduction but recurrence after the patient left the catheter laboratory. Various factors possibly associated with failure or recurrence were analysed, including accessory pathway location, catheter stability, and local electrogram characteristics: QRS-V interval, the AV interval, the presence of a possible accessory pathway potential, the stability of local atrial and ventricular electrograms, the VA interval and time from energy delivery to block of accessory pathway conduction. Accessory pathway location was determined as the site where energy delivery led to interruption of accessory pathway conduction. For four patients in whom energy delivery did not lead to interruption of accessory pathway conduction, the accessory pathway location was

Table 1 Clinical characteristics of patients with primary failure or recurrence of accessory pathway conduction

\begin{tabular}{lcclcl}
\hline & Success & Failure & $P^{*}$ & Recurrence & $P^{\star}$ \\
\hline Mean age (SD) (years) & $34(14)$ & $36(16)$ & $0 \cdot 80$ & $35(16)$ & $0 \cdot 9$ \\
Sex (male/female) & $135 / 70$ & $17 / 8$ & $1 \cdot 00$ & $7 / 6$ & $0 \cdot 56$ \\
Structural heart disease & 6 & 2 & $0 \cdot 43$ & 1 & $0 \cdot 87$ \\
Manifest/concealed AP & $171 / 39$ & $20 / 5$ & $1 \cdot 00$ & $12 / 1$ & $0 \cdot 22$ \\
Multiple AP & 5 & 0 & $0 \cdot 49$ & 0 & $0 \cdot 53$ \\
Number of drug failures & 22 & 21 & $0 \cdot 9$ & 31 & $0 \cdot 21$ \\
Previous EPS & 33 & 5 & $0 \cdot 96$ & 2 & $1 \cdot 00$ \\
\hline AP, accessory pathway; EPS, electrophysiological study. & & &
\end{tabular}

${ }^{\star} \mathrm{P}$ value compared with the success group. determined from the results of endocardial mapping. Misdiagnosis of accessory pathway location was determined when the location of pathways defined in the initial unsuccessful procedure was different from the site where the energy delivery led to a successful ablation of the accessory pathway in a repeat procedure. Unstable catheter position was defined as $50 \%$ or greater variation in amplitude of the electrogram (five complexes immediately preceding radiofrequency current delivery) recorded at the ablation site, or ablation catheter movement from the target site during current delivery, verified by fluoroscopy, or both.

\section{FOLLOW UP}

All patients underwent routine outpatient follow up. If there was a suspicion of recurrence of accessory pathway conduction, an electrophysiological study was performed.

\section{STATISTICAL ANALYSIS}

Continuous variables are expressed as mean (SD). Comparisons between successful and unsuccessful sites were made using the Student's $t$ test or by $\chi^{2}$ analysis. Multivariate stepwise logistic regression was then performed to find the most powerful independent predictors of recurrence. A $P$ value of less than 0.05 was regarded as significant.

\section{Results}

Primary failure of radiofrequency catheter ablation of accessory pathways occurred in 25 of 243 patients $(10 \%)$. Recurrence of accessory pathway conduction occurred in 13 of 218 patients $(6 \%)$ after mean follow up of 20 (13) months (range 6 to 40). Recurrence was manifest by ventricular pre-excitation on routine follow up ECG in eight patients, documented tachycardia in one, and both in four patients. The time to recurrence varied from one hour to within 42 days. In half of these patients recurrence was in the first 12 hours after ablation. In the 35 patients who underwent ablation in 1991, the procedure failed in seven patients and recurrence of accessory pathway conduction occurred in two patients; while in the 208 patients who underwent ablation between 1992 and 1994 primary failure occurred in 18 and recurrence in 11 patients. There were no significant differences in the rate of primary failure and recurrence between 1991 and the following years $(P=0.08$ and $0 \cdot 88$, respectively) although the rate of primary failure seems higher in 1991 . There were no significant differences in the rate of failure or recurrence between manifest and concealed pathways. The clinical characteristics of all patients are summarised in table 1 .

\section{LOCATION OF ACCESSORY PATHWAYS}

Failure of interruption of accessory pathway conduction occurred more frequently in patients with right sided pathways, especially in mid-septal and right anteroseptal locations. Recurrence occurred more often in patients with right free wall pathways (table 2). 
Table 2 Location of accessory pathways

\begin{tabular}{|c|c|c|c|c|c|c|c|}
\hline \multicolumn{2}{|c|}{ Primary failure ${ }^{\star}$} & \multicolumn{2}{|c|}{ Successful ablation } & \multicolumn{2}{|c|}{ Recurrence } & \multicolumn{2}{|c|}{ No recurrence } \\
\hline Location & Patients & Location & Patients & Location & Patients & Location & Patients \\
\hline LFW & 9 & LFW & 118 & LFW & $4 t$ & LFW & 113 \\
\hline LPS & 1 & LPS & 24 & LPS & 2 & LPS & 22 \\
\hline MS & 7 & MS & 23 & MS & 1 & MS & 23 \\
\hline RPS & 3 & RPS & 28 & RPS & 1 & RPS & 27 \\
\hline RAS & 2 & RAS & 9 & RAS & 1 & RAS & 8 \\
\hline RFW & 3 & RFW & 21 & RFW & $4 \dagger$ & RFW & 17 \\
\hline
\end{tabular}

AP, accessory pathway; LFW, left free wall; LPS, left posteroseptal; MS, midseptal; RAS, right anteroseptal; RFW, right free wall; RPS, right posteroseptal.

The failure rate was higher in right sided pathways (MS, RPS, RAS, and RFW as a group) compared with left sided pathways (LFW and LPS as a group) $P<0.05$. The recurrence rate was higher in right free wall compared with left free wall pathways
$(P=0.03)$.

\section{FACTORS ASSOCIATED WITH PRIMARY FAILURE} Unstable catheter position

In patients $2,5,7,8,10,11$, and 17 , an unstable catheter position was the main reason for primary failure (table 3 ). Standard radiofrequency ablation (unipolar technique) was successful at a repeat session in patients $7,8,11$, and 17. In patient 5 a transseptal approach to a left free wall pathway was employed and succeeded. In patient 2 a repeat procedure was not performed, because manipulation of the catheter across the aortic valve and within the left ventricle was extremely difficult due to tortuosity of the iliac vessels. Patient 10 declined a second ablation.

Epicardial accessory pathway? (table 3)

In patients 3, 4, 12, 24, and 25 the best endocardial electrogram was recorded from within the coronary sinus and in patients $3,4,12$, and 25 the delta wave in lead II was strikingly negative. Coronary sinus angiography was performed in patients 12 and 25 and showed a large proximally arising middle cardiac vein in patient 12 and a large cardiac vein and smaller posterior vein in patient 25 . Standard radiofrequency ablation was repeated in patients 3,4 and failed in both; ablation of the atrioventricular junction was performed subsequently. In patient 12 , two further radiofrequency ablation procedures failed and low energy direct current was then delivered in the coronary sinus and ultimately ablated the pathway. In patient 25 repeat standard radiofrequency ablation also failed and low energy direct current delivered between two electrodes positioned in the left ventricle and coronary sinus succeeded in ablating the pathway. Low energy direct current delivered through one electrode in the coronary sinus in this patient caused only transient block. Patient 24 preferred not to have a repeat ablation.

In patient 23 with a right lateral pathway, three procedures using standard radiofrequency ablation technique failed (low energy direct current was used in the third session and also failed) before surgical dissection was undertaken. The accessory pathway was mapped to the right lateral position, and the delta wave disappeared with surgical dissection in the epicardial area overlying the catheter ablation scar.

Successful ablation achieved by low energy direct current or bipolar technique (table 3 )

In patients $1,6,13-15,18-20$, and 22 the factors associated with primary failure were unclear. Repeat standard radiofrequency ablation was attempted in all these patients but failed. In patients 1 and 6 radiofrequency current delivered subsequently in a bipolar fashion across the septum succeeded. In patients $13-15,18-20$, and 22 low energy direct current was employed and succeeded in all

Table 3 Reasons for primary failure

\begin{tabular}{|c|c|c|c|c|c|c|c|c|c|c|c|c|}
\hline Pts & Age/sex & $P x$ & Location & $P T$ & $S T$ & Sites & $U C P$ & $\begin{array}{l}\text { Negative } \\
\text { in II }\end{array}$ & $\begin{array}{l}\text { Best } E G M \\
\text { in } C S\end{array}$ & Other & $\begin{array}{l}\text { 2nd } \\
\text { Ablation }\end{array}$ & Success \\
\hline 1 & $14 \mathrm{M}$ & No & LPS & 180 & 67 & 13 & & & & $M D$ & Bipolar $\neq$ & Yes \\
\hline 2 & $34 \mathrm{M}$ & Yes & MS & 245 & 88 & 23 & Yes & & & & No\# & \\
\hline 3 & $35 \mathrm{~F}$ & Inter & MS & 290 & 96 & 50 & & Yes & Yes & & Unipolar & No, AVN \\
\hline 4 & $71 \mathrm{M}$ & Inter & MS & 360 & 135 & 21 & & Yes & Yes & & Unipolar & No, AVN \\
\hline 5 & $42 \mathrm{M}$ & Yes & LFW & 180 & 56 & 22 & Yes & & & & Transseptal & Yes \\
\hline 6 & $24 \mathrm{~F}$ & Yes & $\overline{M S}$ & 280 & 141 & 17 & & & & & Bipolar & Yes \\
\hline 7 & $42 \mathrm{~F}$ & Inter & RPS & 180 & 52 & 16 & Yes & & & & Unipolar & Yes \\
\hline 8 & $48 \mathrm{M}$ & Yes & LFW & 230 & 89 & 22 & Yes & & & & Unipolar & Yes \\
\hline 9 & $23 \mathrm{M}$ & Yes & RFW & 270 & 105 & 47 & & & & Back pain & Unipolar & Yes \\
\hline 10 & $13 \mathrm{~F}$ & Yes & RAS & 335 & 172 & 49 & Yes & & & & & \\
\hline 11 & $68 \mathrm{~F}$ & No & MS & 240 & 100 & 6 & Yes & & & & Unipolar & Yes \\
\hline 12 & $41 \mathrm{M}$ & Yes & MS & 200 & 125 & 14 & & $\mathrm{Yes}^{\wedge}$ & Yes & & Low energy & Yes \\
\hline 13 & $30 \mathrm{~F}$ & Yes & RAS & 190 & 83 & 29 & & & & & Low energy & Yes \\
\hline 14 & $19 \mathrm{M}$ & Yes & RFW & 225 & 91 & 31 & & & & MD & Low energyt & Yes \\
\hline 15 & $23 \mathrm{M}$ & Yes & LFW & 230 & 86 & 23 & & & & & Low energy & Yes \\
\hline 16 & $42 \mathrm{M}$ & Yes & LFW & 190 & 61 & 21 & & & & & Late block $\oint$ & \\
\hline 17 & $51 \mathrm{~F}$ & No & LFW & 150 & 65 & 10 & Yes & & & & Unipolar & Yes \\
\hline 18 & $33 \mathrm{M}$ & Yes & RPS & 165 & 124 & 32 & & & & & Low energy & Yes \\
\hline 19 & $33 \mathrm{M}$ & Inter & LFW & 103 & 31 & 9 & & & & & Low energy & Yes \\
\hline 20 & $40 \mathrm{~F}$ & Yes & $\overline{R P S}$ & 210 & 72 & 33 & & & & & Low energy & Yes \\
\hline 21 & $39 M$ & Inter & LFW & 90 & 10 & 2 & & & & RAF & Unipolar & Yes \\
\hline 22 & $41 \mathrm{M}$ & Yes & LFW & 160 & 48 & 18 & & & & & Low energy & Yes \\
\hline 23 & $29 \mathrm{~F}$ & No & RFW & 230 & 103 & 39 & & & & & Surgical & Yes \\
\hline 24 & $28 \mathrm{M}$ & No & LFW & 180 & 23 & 9 & & & Yes & & & \\
\hline 25 & $25 \mathrm{M}$ & Yes & MS & 195 & 60 & 20 & & Yes^$^{\wedge}$ & Yes & & Low energy & Yes \\
\hline
\end{tabular}

Ab, ablation; AP, accessory pathway; AVN, successful radiofrequency ablation of atrioventricular node; Best EGM in CS, best endocardial electrogram obtained within coronary sinus; F, female; Inter, intermittent pre-excitation; LFW, left free wall; LPS, left posteroseptal; M, male; MD, misdiagnosis of accessory pathway location; MS, mid-septal; Negative in II, strikingly negative delta wave in lead II; PT, procedure time; Pts, patients; Px, pre-excitation; RAF, recurrent atrial fibrillation during procedure; RAS, right anteroseptal; RFW, right free wall; RPS, right posteroseptal; Sites, number of sites at which energy was delivered; ST, screening time; UCP, unstable catheter position.

†Right posteroseptal location diagnosed in the first procedure, actual location (right free wall) identified in repeat successful procedure in which conventional unipolar radiofrequency current was attempted prior to low energy ablation.

¥Midseptal location diagnosed in first procedure, left posteroseptal position identified in repeat successful procedure in which conventional unipolar radiofrequency ablation was attempted prior to successful ablation using bipolar technique.

§Late permanent block following first radiofrequency ablation.

\#Manipulation of the catheter across the aortic valve and within the left ventricle was extremely difficult due to tortuosity of the iliac vessels.

${ }^{\wedge}$ Coronary sinus angiography revealed a large middle cardiac vein. 
Table 4 Final success with low energy direct current shock or bipolar radiofrequency technique

\begin{tabular}{|c|c|c|c|c|c|c|c|c|}
\hline & $\begin{array}{l}\text { Initial } \\
R F\end{array}$ & Sites & $\begin{array}{l}\text { Transient } \\
\text { block }\end{array}$ & $\begin{array}{l}\text { Same } \\
\text { site }\end{array}$ & $\begin{array}{l}\text { Initial shock } \\
\text { (joules) }\end{array}$ & $\begin{array}{l}\text { Total energy } \\
\text { (joules) }\end{array}$ & $\begin{array}{l}\text { Prior } \\
R F\end{array}$ & $\begin{array}{l}\text { Low energy } \\
\text { success }\end{array}$ \\
\hline 12 & Yes & 6 & No & Yes & 5 & 92 & 3 & Yes \\
\hline 13 & Yes & 29 & 4 & No & 20 & 310 & 1 & Yes \\
\hline 14 & Yes & 18 & 1 & Yes & 10 & 190 & 2 & Yes \\
\hline 15 & Yes & 23 & 6 & Yes & 5 & 85 & 1 & Yes \\
\hline 18 & Yes & 7 & 2 & Yes & 5 & 115 & 1 & Yes \\
\hline 19 & Yes & 9 & 4 & Yes & 5 & 90 & 1 & Yes \\
\hline 20 & Yes & 17 & No & Yes & 2 & 132 & 1 & Yes \\
\hline 22 & Yes & 18 & 1 & Yes & 2 & 172 & 1 & Yes \\
\hline 25 & Yes & 6 & No & No & 20 & 222 & 2 & Yes \\
\hline & Initial RF & Site & Transient block & & & & & Bipolar success \\
\hline 1 & Yes & 17 & No & Yes & & & & Yes \\
\hline 6 & Yes & 1 & No & Yes & & & 2 & Yes \\
\hline
\end{tabular}

Initial RF, standard radiofrequency ablation was attempted before low energy direct current or bipolar radiofrequency technique; Initial shock, magnitude of low energy direct current shock causing interruption of accessory pathway conduction; Prior RF, number of failed radio frequency ablation sessions before the successful session; Same site, successful low energy ablation at site where radiofrequency ablation failed; Sites, number of radiofrequency current delivery sites; Transient block, number of
episodes of transient block of accessory pathway conduction during radiofrequency ablation.

patients. In patients $14,15,18-20$, and 22 low energy direct current was successful in abolishing accessory pathway conduction when delivered at the same site where standard unipolar radiofrequency current had failed (table 4).

Other factors (table 3)

In patients 1 and 14 misdiagnosis of accessory pathway location occurred during the first procedure. In patient 21 , recurrent atrial fibrillation together with intermittent anterograde conduction in the accessory pathway during the procedure contributed to failure. In patient 9 the procedure failed because of patient discomfort (back pain) precluding continuation under local anaesthesia. In patient 16 , late block of accessory pathway conduction was observed.

The median procedure and fluoroscopic times were longer $(180 v 80$ minutes, $\mathrm{P}<$ $0.05 ; 65 \vee 21$ minutes, $P<0.05$ ) and the number of radiofrequency ablation sites was greater $(18 v 5, \mathrm{P}<0.05)$ in the failed group compared with the successful group.

SUMMARY

In summary, repeat catheter ablation was performed in 20 patients and succeeded in 18 . Standard radiofrequency ablation technique was initially used in all patients in the repeat procedure, two patients subsequently underwent successful bipolar radiofrequency ablation, and nine patients underwent low energy direct current ablation (table 4). After a mean follow up period of 14 (11) months (range 3 to 34) there was no recurrence. No difference in QRS-V interval (-5.6 (5) $v-5 \cdot 7(7 \cdot 9) \mathrm{ms})$ and $\mathrm{AV}$ interval (30 (10) v 34 (9) $\mathrm{ms})$ was observed between patients during the initial failure and the repeat successful procedure. In the two patients with concealed accessory pathways in whom a repeat procedure succeeded after primary failure, the VA interval recorded on the ablating catheter was shorter on the second occasion ( 30 and $40 \mathrm{~ms} v 45$ and $50 \mathrm{~ms}$, respectively).

\section{RECURRENCE OF ACCESSORY PATHWAY} CONDUCTION

The time from onset of radiofrequency current to block of accessory pathway conduction was $\geqslant 12$ seconds $(12,14.5,27,86)$ in four of 13 patients with recurrence compared to eight of 205 patients without recurrence $(\mathrm{P}=$ $0.0005)$. In one of the four, temperature monitoring was used during delivery and the desired temperature could not be constantly achieved despite maximum power output (50 W).

Recurrence of accessory pathway conduction occurred in five of eight patients with poor ablation electrogram stability (atrial stability $\leqslant 0.5$, ventricular stability $\leqslant 0 \cdot 8$ ) compared to eight of 210 patients with stable ablating electrograms $(P=0.001)$.

Multivariate stepwise logistic regression showed that prolonged time to block ( $P=$ 0.003 ) and stability of the local atrial electrogram $\leqslant 0.5$ together with stability of the local ventricular electrogram $\leqslant 0.8 \quad(P=0.002)$ were independent predictors of recurrence.

The median procedure and fluoroscopic times were longer (162 v 80 minutes $(\mathrm{P}<$ $0.05)$ and $47 v 21$ minutes $(P<0.05))$ and the number of radiofrequency ablation sites was greater $(18 v 5(\mathrm{P}<0.05))$ in the group with recurrent conduction than in that without.

No differences were observed in the presence of transient block of accessory pathway conduction before interruption of accessory pathway conduction $(4 / 48$ v $8 / 170 \quad(\mathrm{P}=$ $0.54)$ ), presence of a possible accessory pathway potential $(5 / 13 v 55 / 205(\mathrm{P}>0.05))$, QRS-V interval $(-11.6(13.8) v-9.5(12.3)$ ms (P > 0.05)), AV interval (34 (18) v 31 (12) $\mathrm{ms}(\mathrm{P}>0.05))$, between recurrence and nonrecurrence groups.

Repeat ablation was performed in 11 patients (and scheduled in the remaining two) and succeeded in 10. Standard radiofrequency ablation technique was initially employed during the repeat procedure in all patients and succeeded in seven. In the remaining three patients, low energy direct current ablation (two patients) and bipolar ablation (one patient) succeeded at the same site where standard unipolar radiofrequency energy initially failed. There was no further recurrence after mean follow up 12 (11) months (range 2 to 25). No differences in QRS-V interval ( -11 (13.6) $v-11(8 \cdot 6) \mathrm{ms}(\mathrm{P}>0.05))$, AV interval (41 (23) v 37 (19) $\mathrm{ms}(\mathrm{P}>0.05)$ ), the presence of a possible accessory pathway potential 
$(3 / 12 v 2 / 11(P>0.05))$, and the power of the radiofrequency application for ablation $(32$ (10) $v 33(9) \mathrm{W}(\mathrm{P}>0.05)$ were observed between the two procedures, with the exception of time to block, which was longer in the initial procedure $(4.5(4.6)$ v $2.4(1.4)(\mathrm{P}<$ 0.01 ); the patient with the longest time to block (86 seconds) was not included in the statistical analysis).

\section{Discussion \\ FAILURE OF INTERRUPTION OF ACCESSORY PATHWAY CONDUCTION}

Failure to abolish accessory pathway conduction in a single procedure occurred in $10 \%$ of patients. In seven of the 25 patients an unstable catheter position was identified as the main reason for primary failure of accessory pathway ablation. This may have been due to individual anatomical variation, catheter design, mode of approach to the atrioventricular valve annulus, rhythm during energy application, the use or not of a supporting long sheath, or as yet unidentified factors. However, in these patients, a repeat radiofrequency ablation procedure often led to successful interruption of accessory pathway conduction.

In five patients the best endocardial electrogram was recorded from within the coronary sinus and in four patients the delta wave in lead II was strikingly negative. Arruda et $\mathrm{al}^{5}$ studied 255 patients (135 retrospectively and 120 prospectively) undergoing radiofrequency ablation of accessory pathways and showed that posteroseptal accessory pathways in an epicardial location or with venous anomalies can be identified by a strikingly negative delta wave in lead II (false positive rate $<1 \%$ ). In those patients, radiofrequency energy applied at usual posteroseptal ablation sites was unsuccessful. In the present study, one or more standard radiofrequency ablation sessions were performed in four patients and failed in all (in two, coronary sinus angiography showed a large middle cardiac vein, and low energy direct current delivered in the coronary sinus led to success). This result, together with the surface ECG and endocardial electrogram features, favours an epicardial pathway. In one patient (in whom three radiofrequency ablation sessions failed and in whom low energy direct current in the final procedure also failed) this was proven when an epicardial pathway was confirmed at surgical dissection.

In nine patients repeat standard radiofrequency ablation was attempted but failed. In two of these, radiofrequency current delivered in bipolar fashion across the septum was subsequently successful. In another seven patients, low energy direct current was employed and succeeded in all, four of whom had successful low energy direct current deliveries at the same site where radiofrequency energy failed. The reason why standard unipolar radiofrequency ablation failed and bipolar radiofrequency delivery or low energy direct current succeeded at the same site in some patients is unclear. Previous studies have shown that the lesion produced by low energy direct current and bipolar radiofrequency energy is larger than that by standard unipolar radiofrequency energy.$^{6-9}$ In these cases we concluded that the catheter could not be placed close enough to the accessory pathway or that anatomical variation of the accessory pathway may have played a role. The small lesions created by standard radiofrequency current could not penetrate to the pathway, or caused only transient or partial block. Low energy direct current shocks or bipolar radiofrequency energy delivery may, by creating a larger and deeper lesion, permit a successful outcome in these cases.

The accessory pathway location was incorrectly identified in two patients. Following improvements in mapping technique this is not a common reason for failure. Recurrent atrial fibrillation in conjunction with intermittent anterograde conduction through the accessory pathway may occasionally make ablation difficult.

\section{RECURRENCE OF ACCESSORY PATHWAY} CONDUCTION

Recurrence of accessory pathway conduction occurred in $6 \%$ of patients. Langberg et al ${ }^{10}$ reported a $12 \%$ recurrence rate in their series and Twidale et $a l^{11}$ reported $8 \%$. Independent factors which predicted recurrence in our series included poor ablating electrogram stability and prolonged time to block $\geqslant 12 \mathrm{sec}$ onds). It has previously been reported that time to block is longer in accessory pathways which later show recurrence of conduction, suggesting either electrode-tissue contact or lack of proximity of the ablating electrode to the pathway. ${ }^{11}$ Either temperature monitoring or continuous impedance monitoring may provide important verification of tissue-electrode contact, and previous studies have suggested that catheter tip temperature monitoring may be useful for detecting poor electrodetissue contact. ${ }^{12} 13$ The principal mechanism of radiofrequency induced myocardial injury is presumed to be thermal, with cell injury and death reproducibly occurring once the temperature reaches $50^{\circ} \mathrm{C}$. The lesion size is proportional to the temperature at the electrodetissue interface. If power is applied and little or no heating is observed, poor electrode-tissue contact is suggested. In one of our patients with prolonged time to block of accessory pathway conduction, temperature monitoring showed the target temperature could not be achieved despite maximum power output (50 W).

\section{LOCATION OF ACCESSORY PATHWAYS}

Failure to ablate and recurrent conduction occurred more frequently in right sided accessory pathways, consistent with previous studies. $^{21011}$

LOCAL ENDOCARDIAL ELECTROGRAM

There were no differences in QRS-V interval, AV interval, VA interval, and the presence of a possible accessory pathway potential between the initial procedure and the repeat successful 
procedure in patients with primary failure. In patients with recurrence, the time to block was longer in the initial procedure, as in previous reports. An earlier study also suggests that the absence of accessory pathway potential is a strong predictor for recurrence of accessory pathway conduction. ${ }^{11}$ In our series, as is common practice in most centres, possible accessory pathway potentials were noted but no attempt was made at verification.

\section{CONCLUSIONS}

Failure of ablation and recurrence of accessory pathway conduction were more frequent in patients with right sided accessory pathways. Catheter instability and possible epicardial location were identified as the major reasons for primary failure. Where catheter instability is identified then there is a good chance of success with a further attempt, but pathways situated deep within the myocardium may well require an alternative method of energy delivery, in particular the use of bipolar radiofrequency energy delivery or low energy dc shocks should be considered.

Where recurrence of accessory pathway conduction occurs after initially successful ablation, catheter instability or prolonged time to conduction block during energy delivery were identified as the likely cause. This, once again, emphasises the need for stable catheter contact and precise mapping to position the ablation electrode as close as possible to the substrate for ablation.

1 Jackman WM, Wang X, Friday KJ, Roman CA, Moulton $\mathrm{KP}$, Beckman KJ, et al. Catheter ablation of accessory atrioventricular pathways (Wolff-Parkinson-White syn- drome) by radiofrequency current. $N$ Engl f Med 1991; 324:1605-11

2 Lesh MD, Van Hare GF, Schamp DJ, Chien W, Lee MA Griffin JC, et al. Curative percutaneous catheter ablation using radiofrequency energy for accessory pathways in all using radiofrequency energy for accessory pathways in all locations: results in 100

3 Bashir Y, Heald SC, Katrisis D, Hammouda M, Camm AJ, Ward DE. Radiofrequency ablation of accessory atrioventricular pathways: predictive value of local electrogram characteristics for the identification of successful target sites. Br Heart f 1993;69:315-21.

4 Ward DE, Camm AJ, Pearce RC, Spurrell RAJ, Rees GM Incessant atrioventricular tachycardia involving an accessory pathway: preoperative and Intraoperative electrophysiological studies and surgical correction. $\mathrm{Am}$ Cardiol 1979;44:428-34

5 Arruda MS, Wang X, McClelland JH, Beckman KJ, Nakagawa $\mathrm{H}$, Widman LE, et al. Negative delta wave in lead II identifies posteroseptal accessory pathways requiring ablation in venous branches of the coronary sinus [abstract]. $\mathcal{F} \mathrm{Am}$ Coll Cardiol 1994;73:224A.

6 Katritsis D. Heald S, Bashir Y, Ward DE, Rowland E. Low energy ablation of accessory pathways in cases for which the radiofrequency

7 Lemery R, Talaiic M, Roy D, Fournier A, Coutu B, Hiij T, et al. Catheter ablation using radiofrequency or low energy direct current in paediatric patients with the Wolff-Parkinson-White syndrome. Am $\mathcal{F}$ Cardiol 1994;73 191-4

8 Jackman WM, Kuck KH, Naccarelli GV, Carmen L, Pitha J. Radiofrequency current directed across the mitral annulus with a bipolar epicardial-endocardial catheter electrode configuration in dogs. Circulation 1988;78 1288-98.

9 Bashir Y, Heald SC, O'Nunain S, Katritsis D, Camm AJ, Ward DE. Radiofrequency current delivery by way of a bipolar tricuspid annulus-mitral annulus electrode configuration for ablation of posteroseptal accessory pathways. F Am Coll Cardiol 1993;22:550-6.

10 Langberg JJ, Calkins H, Kim YN, Sousa J, El-Atassi R, Leon A, et al. Recurrence of conduction in accessory atrioventricular connections after initially successful atriA frequency

11 Twidale $\mathrm{N}$, Wang $\mathrm{X}$, Beckman $\mathrm{KJ}$, McClelland $\mathrm{JH}$, Moulton KP, Prior MI, et al. Factors associated with recurrence of accessory pathway conduction after radiorecurrence of accessory pathway conduction after radio

12 Haines DE. The biophysics of radiofrequency catheter ablation in the heart: the importance of temperature ablation in the heart: the importanc

13 Avitall B, Khan M, Krum D, Hare J, Lessila C, Dhala A, $e t$ al. Physics and engineering of transcatheter cardiac tissue ablation. $₹$ Am Coll Cardiol 1993;22:921-32. 